\title{
TOMA DE DECISIÓN E IMPLANTACIÓN EN LA POLÍTICA AGROAMBIENTAL ESPAÑOLA
}

\author{
POR \\ ÁNGEL PANIAGUA MAZORRA
}

\section{Introducción}

Desde mediados de los años ochenta, la agricultura europea adopta una orientación más ambiental en la que influyen, además de problemas ecológicos y presupuestarios, una serie de transformaciones de tipo socioeconómico, demográfico y de actitudes ambientales de la población (Ilbery, 1992) ${ }^{1}$. Todo ello da lugar a modificaciones institucionales y a la aparición de nuevos instrumentos de intervención en el sector agrario sobre todo a partir de la reforma de la Política Agraria Común (PAC) de 1992 (Baldock, Lowe, 1996; Deverre, 1995; Guglielmi, 1995). Se ha apuntado que una de las principales características de la introducción de medidas de reforma de la PAC es su desarrollo institucional y político a diversos niveles de decisión, especialmente nacional y regional, sobre todo en las medidas de acompañamiento (Lowe, Ward, 1998). Una de estas medidas, la normativa agroambiental comunitaria, establece que cada Estado miembro puede determinar sus propias zonas sensibles, las prácticas de producción compatibles con las exigencias de la protección del medio ambiente y las normas y criterios de las prácticas de pro-

${ }^{1}$ Este artículo forma parte del proyecto de investigación PB95-0076 financiado por la DGES.

Ángel Paniagua Mazorra: Instituto de Economía y Geografía, CSIC. Madrid.

Estudios Geográficos

Tomo LXI, 2000, n. ${ }^{\circ} 239$, abril-junio 
ducción (Wilson, 1995). La aplicación del principio de subsidiariedad ha provocado que cada país, e incluso cada región, adopte regulaciones agroambientales extremadamente variadas de acuerdo con sus intereses (Wilson, 1995; Potter, 1998). De esta forma es posible configurar estilos nacionales en la toma de decisiones sobre política agroambiental (Clark et al., 1997; Clark, Jones, 1998), fundados en la combinación de distintos factores entre los que se han señalado: la percepción social de la agricultura en cada país; la construcción social de los problemas ambientales rurales y la responsabilidad de la agricultura en su generación; la importancia del sector agrario en la toma de decisión regional y local; la identidad rural nacional, y los estilos institucionales generados en el sector agrario en cada nación (Hoggart, Buller, Black, 1995; Buller, 1992).

El desarrollo de prácticas agrarias respetuosas con el medio ambiente constituye un elemento esencial de la política agraria de los años noventa (Battershill, Gilg, 1997: 213). Su efectiva implantación, dado su carácter voluntario, depende del interés de los agricultores en participar o no participar de acuerdo a sus propios intereses instrumentales, valores y costumbres y, en consecuencia, pueden reordenar las prioridades establecidas en niveles de toma de decisión superior (Curtis, De Lacey, 1998; Ward et al., 1998; Murdoch, Ward, 1997; Ward et al., 1998).

De esta forma en cada escala o nivel de adopción de decisiones sobre la política agroambiental (nacional, regional y local o de implantación) es factible reordenar y reorientar las prioridades sobre la regulación agroambiental de acuerdo a la percepción de cómo afectan a los intereses de cada actor social dentro del contexto decisional en el que se desenvuelve. En el proceso escalonado de diferenciación de la política agroambiental, los países del sur de Europa constituyen un adecuado ejemplo, dado que presentan unas claras especifidades que hacen extremadamente compleja la puesta en práctica de medidas de carácter ambiental en la agricultura respecto a otros del norte, en buena parte debido a que el balance entre la protección ambiental y la renta de explotación es extremadamente delicado (Potter, Goodwin, 1998).

En España, junto a factores de cambio que, con mayor o menor intensidad, son comunes a todos los países europeos (modificación en las pautas de consumo alimentario, mayor demanda de servicios ambientales de las zonas rurales, incremento de la renta de los hogares agrarios...), el proceso de ambientalización de la agricultura española es sin- 
gular debido a distintas características, entre las que destacan: la propia originalidad de los problemas ambientales rurales; el menor grado de intensificación de la agricultura respecto a otros países centroeuropeos; la creciente, pero aún débil, influencia de nuevos actores sociales en la política agraria; y los acusados problemas de despoblación en áreas rurales del interior. Además, España, por su compleja distribución de competencias administrativas agrarias y ambientales entre el Estado y las Comunidades Autónomas (CCAA), acusadamente descentralizada, permite analizar la generación de estilos de implantación de políticas agroambientales regionales (Garrido, Moyano, 1996, Paniagua, 1997, Paniagua et al., 1998).

Este artículo pretende explorar los discursos y estrategias de distintos actores con influencia en la adopción de decisiones en el desarrollo de la política agroambiental a diversas escalas o niveles (nacional, regional y local o de implantación) en el contexto del proceso de toma de decisión e implantación de la regulación agroambiental en España. Los actores implicados pueden promover discursos ambientales variables, con múltiples facetas, en la adopción de decisiones, que pueden referirse tanto a cuestiones y prácticas específicas de la gestión ambiental, como estar asociadas a aspectos más básicos (ej.: la propia interacción hombre-medio ambiente) y que, en su conjunto, generan un potencial incertidumbre sociocultural en la gestión ambiental (Wilson, Bryant, 1997; Billaud et al., 1997).

La selección de áreas de estudio y el diseño del trabajo de campo. El trabajo de campo ha constado de entrevistas semiestructuradas ${ }^{2}$ a decisores públicos, representantes de sindicatos agrarios y representantes de grupos ecologistas. Además de tres grupos de discusión, de distintos perfiles, con agricultores. El guión de los grupos constaba de tres grandes partes: en primer lugar se trataba de buscar una definición de naturaleza, medio ambiente o problema ambiental; en segundo lugar, establecer los conflictos entre la percepción del medio ambiente y la

2 El número de entrevistas realizadas asciende a 43. El guión utilizado en las entrevistas es el siguiente: definición de medioambiente; opinión sobre la armonización de los esfuerzos entre la administración europea, estatal y autonómica en la trasposición e implantación de medidas agroambientales; participación e integración de diversos grupos sociales en la confección de las regulaciones agroambientales; relaciones con la administración de otros grupos sociales o entre diversas administraciones; no productivismo; armonía sistemas agrarios/ecosistemas; agricultura sostenible/sustentable; el pacto social agricultores-sociedad. 
profesión de agricultor; en tercer lugar y, por último, se intentaba establecer la percepción de los programas agroambientales en relación a la práctica profesional de cada zona. Los tres grupos de discusión se realizaron en Castilla y León, constituidos, cada uno, de acuerdo con las características más relevantes de su zona:

1. Ubicado en Osorno (Palencia). Área de páramo y agricultura convencional. Constituido por agricultores menores de 45 años o mayores pero con hijos sucesores trabajando en la explotación; el tamaño de la explotación medio-grande (a partir de 100-150 ha); con orientación productiva de regadío extensivo; y, por último, con y sin formación agraria especializada.

2. Ubicado en Santa María la Real de Nieva (Segovia). Área de campiña y agricultura más tradicional. Constituido por propietarios mayores de 50 años, algunos en edad de retiro, sin sucesor claro; con una orientación productiva tradicional, explotación de tamaño reducido, explotación muy parcelada, sin formación reglada.

3. Ubicado en Villarín de Campos (Zamora). Grupo en el cual se buscaba enfrentar dos perspectivas: una orientación productiva más moderna frente a otra más tradicional, así como conocer las diferencias de cada una respecto a los contratos agroambientales. El perfil del grupo es de agricultores con y sin contrato agroambiental; orientación productiva tradicional y moderna; propietarios entre 40 y 60 años; tamaño de las explotaciones medio-alto (alrededor de las $100 \mathrm{ha}$ ); con y sin formación agraria especializada.

El diseño metodológico trataba de establecer los discursos y estrategias en relación al proceso de implantación de la política agroambiental a tres niveles: nivel nacional, regional y local o de implantación. Con ello se pretendía explorar la consistencia entre los distintos niveles o escalas de la toma de decisión y entre zonas u áreas geográficas dentro del nivel regional o de implantación. Con este fin se realizó un análisis a nivel nacional y otro a nivel regional en Castilla y León y dentro de esta CA, en tres zonas diferentes (por su orientación productiva y características de la agricultura( afectadas por medidas de carácter agroambiental. La CA de Castilla y León se escogió al desarrollarse en la misma el pionero - se inicia en 1993- Programa de Estepas Cerealistas, que es el de mayor extensión y de mayor fundamento en razones ex- 
clusivamente agroambientales de este tipo llevado a cabo en España. Se calcula que en el área vive el 54\% de la población de avutardas de España y el 26\% de la mundial (Programa, 1992).

\section{La política agroambiental a nivel nacional}

En España, la regulación ambiental en la agricultura ha sido escasa (Paniagua, 1997). Previamente a la normativa agroambiental de la Unión Europea prácticamente no existe. Los esfuerzos específicos para introducir requerimientos ambientales en las prácticas agrícolas por la $A d-$ ministración Agraria, se producen en una etapa de cambio político. Esto último queda adecuadamente reflejado en la estrategia integrada del Ministerio de Agricultura para la conservación de la naturaleza de 1995 (ENCINA). Este programa presenta un carácter rural e intenta establecer un marco de referencia para la actividad del Ministerio de Agricultura y los actores sociales del sector. El documento, que adopta el ideario ambientalista de los países occidentales, pretende orientar las actividades de los profesionales en el sector y de la administración ambiental para preservar los recursos no renovables. Fue elaborado bajo el gobierno socialista, pero no se implanta debido a que después del triunfo del centro-derecha en 1996, el sistema de parques nacionales y espacios naturales, principal instrumento con el que constaba, se transfirió al nuevo Ministerio de Medio Ambiente. No obstante, el nuevo gobierno mantiene un punto de vista sobre la agricultura sustentable que combina aspectos de la ética implícita a la agricultura tradicional junto a un cierto nacionalismo rural, que queda asociado a la preservación del paisaje y los valores rurales, combinado con la introducción de mejoras tecnológicas. Los instrumentos de intervención se circunscriben a las medidas agroambientales con las que se pretende un desarrollo sostenible que armonice las funciones productiva, social y ecológica del agricultor.

La administración pública española agraria presenta un único discurso respecto a la política agroambiental. Las medidas agroambientales de introducen y desarrollan en España por la pertenencia a un espacio geopolítico de mayor amplitud: la UE. Son medidas diseñadas para los países del centro y norte de Europa y no están adptadas a los problemas de las áreas mediterráneas. Para el 'usuario urbano del campo' 
los puntos de conflicto entre la actividad agrícola y el medio ambiente son imperceptibles. En este contexto, para la Administración no existe una presión de la opinión pública que aconseje la introducción de las medidas agroambientales. A la vez, se plantea un cierto escepticismo sobre las actuales medidas agroambientales, de las que se duda de su eficacia ambiental, al constituir un simple mosaico, fruto de los intereses, descoordinados e inclusocontradictorios, de las distintas CCAA. Las medidas zonales propuestas por cada gobierno autónomo obedecen al juego de intereses propio entre diversos agentes sociales de cada región, en ciertos casos en menoscabo de su finalidad agroambiental. Desde la administración estatal agraria se advierte que, dado el peso de las medidas zonales en el programa agroambiental español, éste constituye un 'mosaico' con escasa conexión entre los programas de las distintas autonomías, lo que limita su efectividad ambiental. El punto de vista regional ha prevalecido sobre el nacional en la preparación del programa agroambiental, lo que se podría haber neutralizado incorporando sus medidas a las Organizaciones Comunes de Mercado (OCMs). Por otra parte, también se plantea que los problemas en la implantación de medidas agroambientales asociados al escaso presupuesto, fruto de su carácter cofinanciado y al desigual interés que presentan para los distintos actores sociales, dado que se percibe que únicamente son relevantes para las asociaciones conservacionistas (en particular la Sociedad Española de Ornitología) y para los sindicatos de izquierdas.

Sindicatos agrarios y medio ambiente en España. Desde hace algunos años los sindicatos agrarios integran la preocupación ambiental en su estrategia de actuación e incluso cambian sus estructuras organizativas para conseguir una respuesta más adecuada a sus preocupaciones ambientales. Con el fin de examinar la representación de problemas ambientales entre los sindicatos agrarios se han seleccionado sus opciones organizativas: la Asociación Sindical de Jóvenes Agricultores (ASAJA), que representa el modelo empresarial; y al Unión de Pequeños Agricultores (UPA), que constituye la izquierda del sindicalismo agrario, con un discurso marcadamente neorrural (Paniagua, 1997). Sus principales puntos de coincidencia son: en primer lugar, que la imposición de la función de 'conservador ambiental' entre los agriculotres debe ir acompañada de nuevas fuentes de ingresos, que puedan reemplazar la pérdida de ingresos causadas por la extensificación y limitación de su ac- 
tividad productiva. En definitiva supone una renegociación del contrato social implícito establecido en el Tratado de Roma, configurador de la UE. Tal alteración en la naturaleza de los subsidios de los agricultores es contemplada por los sindicatos agrarios como un cambio cualitativo en el contrato social que los agricultores mantienen con el resto de la sociedad. En segundo lugar, se señala (con diversas características entre los diferentes sindicatos sobre su origen, agentes involucrados, naturaleza y extensión) la existencia de serios problemas ambientales que pueden afectar a la organización de la actividad agraria.

Pero, los sindicatos agrarios también mantienen diferentes aproximaciones a cuestiones ambientales concretas. Los sindicatos de corte empresarial plantean que el deterioro producido por los agricultores está inducido por el consumo de masas de nuestra sociedad urbana. Los últimos responsables del deterioro del medio ambiente rural son los habitantes de las ciudades que demandan una producción masiva y con precios razonables. En general, se advierte una actitud crítica respecto a las medidas que desincentivan la producción.

En contraste, organizaciones agrícolas de 'izquierdas' entienden que un modelo de agricultura más ecológica puede incrementar los ingresos de los pequeños agricultores sobre la producción de calidad, especialmente en las áreas más marginales. La introducción de medidas agroambientales puede contribuir a mantener un nivel de población adecuado en las áreas rurales, como parte de una estrategia de desarrollo rural.

Concretamente, en relación a la implantación de programas agroambientales, los factores que influencian el limitado proceso de implantación, según las OPAs son: las ayudas no van dirigidas sólo a agricultores profesionales; el excesivo tiempo de duración de los contratos; la rigidez del calendario de las prácticas agrícolas; y, finalmente, la competencia respecto a otras ayudas de la PAC.

El proceso de implantación del programa agroambiental espanol. Entre las nuevas medidas de acompañamiento de la PAC, la agroambiental es la última en trasponerse. Supone el reconocimiento institucional de la nueva función ambiental de los agricultores en beneficio de la sociedad, por la que deben ser remunerados (Secretaría General de Estructuras Agrarias, 1994). El punto de vista inicial -1991— del Ministerio de Agricultura en la negociación sobre las medidas agroambientales pretendía que éstas constituyeran un complemento a los in- 
gresos de los agricultores, antes que interpretarlas como políticas en favor del medio ambiente: «Estas medidas (las agroambientales), que en principio recibirán más apoyo que las demás estaría destinada a ser el eje de la reforma en el sentido de servir de comodín para complementar las rentas de los agricultores. Se trata, así, de una medida de definición amplia donde podrían caber conceptos muy distintos» (MAPA, Gabinete Ministro, 1991, p. 36). Sin embargo, en el propio proceso de negociación entre la Comisión y los estados miembros, entre 1991 y 1992, se impone un sentido más restrictivo al deseable por la administración española, introduciendo la exclusividad de la función ambiental en las medidas agroambientales: «Se adaptarán las propuestas de la Comisión relativas a las medidas de acompañamiento con el fin de tomar en cuenta las siguientes modificaciones (...) El régimen de ayudas se destinará exclusivamente a compensar las medidas que tengan un efecto positivo para el medio ambiente» (cursiva propia) (Ministerio de Agricultura. Leg. 12009. Gabinete del Ministro, p. 14).

Al mismo tiempo, el propósito inicial de la administración agraria estatal consistía en la creación de unas bases nacionales de coordinación con un cierto, pero limitado, desarrollo posterior en cada región. En el proceso de negociación del programa agroambiental español entre el gobierno y las CCAA, y posteriormente en su implantación prevalecen los intereses de las diferentes regiones.

En este contexto no sorprende el complejo camino seguido durante la aprobación del programa agroambiental. En todo caso, España es el país miembro que presenta un mayor número de programas agroambientales en la UE, en total 66, tres horizontales y el resto zonales, en áreas geográficas determinadas. Por esta razón, entre las propuestas regionales de carácter zonal existen diversos objetivos y puntos de vista sobre la actuación agroambiental (Regina Segura, 1996). También se puede apuntar que, en la generación del programa agroambiental, existe una escasa participación de las OPAs y de las organizaciones ambientales no gubernamentales (Paniagua, 1997).

$\mathrm{Al}$ final de este proceso de negociación, las medidas agroambientales se dividen en dos grandes tipos: un programa horizontal, aplicable al conjunto del país, adoptado en septiembre de 1994 y de un programa por áreas o zonal, aprobado en 1995, con facetas operativas: por una parte, los parques nacionales y las áreas sensibles y, por otra, las áreas de interés ecológico definidas por cada Comunidad Autónoma. En su 
conjunto, el programa agroambiental español fue adoptado por el comité STAR de la UE en enero de 1995. Después del gran número de programas sometidos a evaluación, sólo dos, además de los horizontales, se habían puesto en marcha en 1995. En este año, los programas iniciados en Francia y Alemania abarcaban más de 5 millones de ha; en España era de 89.802 ha y en Portugal ascendía a 471.312 ha (en Gran Bretaña ascendía a 796.219 ha). Por tanto, en España el proceso de puesta en marcha de los programas agroambientales es mucho más reducido que en otros países de la UE (Comisión Europea, 1996).

Los fines del programa horizontal son limitados respecto a los propuestos por la regulación UE 2078/92. De los siete tipos de medidas propuestas en la regulación comunitaria sólo se trasponen dos, la reducción en el uso de fertilizantes y productos que puedan afectar a las plantas y la utilización de prácticas de producción que respeten el medio ambiente. Las medidas adoptadas son las que tienen mayor relación con la dinámica agrícola. El resto se incluyen de una u otra forma en los programas regionales. Los dos tipos de acciones horizontales seleccionadas respecto de las comunitarias, por el gobierno español, se desarrollan en cuatro tipos de ayudas horizontales: el fomento de la producción extensiva de cereal, la promoción de la agricultura biológica, el mantenimiento de razas en peligro de extinción y, el desarrollo de programas de formación ambiental. La aplicación de este programa se calculaba que afectaría a medio millón de agricultores y a 3,5 millones de ha. De las medidas citadas, la extensificación de áreas cerealistas se esperaba que recibiera 8 de cada 10 ayudas.

Las medidas horizontales deben ser traspuestas y reguladas por cada Comunidad Autónoma, desde la normativa nacional. En la primera fase del programa (1993-1997), todas las CCAA, excepto Cantabria, habían regulado las medidas horizontales, aunque con distinto ritmo e intensidad, de acuerdo al interés que presentaban para cada política agraria regional. Las CA con gran extensión de sistemas de cereal extensivo - Castilla-La Mancha y Castilla-León- las implantan con celeridad, al constituir una fuente de renta complementaria o alternativa para una buena parte de sus agricultores. Otras, como por ejemplo Cantabria, no las han aplicado, principalmente por no encontrarse representados sus objetivos de política agraria regional, dirigida hacia la especialización de producción láctea. De esta manera, a finales de 1998 se habían invertido 6.003 millones de pesetas en el programa agroam- 
biental horizontal (3.284 millones en extensificación de zonas cerealistas, 1.400 millones en agricultura ecológica, 524 millones en mantenimiento de razas en peligro de extinción y 727 en formación agroambiental). Estas cifras suponen un 8,5\% de las previstas inicialmente y ponen de relieve las dificultades políticas y administrativas del proceso de implantación del programa agroambiental.

Las medidas zonales de iniciativa regional en el programa agroambiental. Las áreas seleccionadas están dominadas por las que tienen una designación de área natural y por la lista propuesta por cada CA. El objetivo común de estas medidas zonales es suplementar y diversificar los ingresos de los agricultores con el fin de mantener sus ingresos y, al mismo tiempo, asegurar unas prácticas ambientales respetuosas del medio ambiente. Ello confiere al programa un carácter rural, que combina factores ecológicos, ambientales, culturales, demográficos, de uso del suelo, agrarios... Están dominados por la iniciativa particular de cada región. La desigual puesta en marcha de las medidas zonales, dependiendo del interés y la capacidad financiera de cada CA corrobora esta interpretación.

Entre 1993 y 1997 sólo seis programas zonales fueron desarrollados e implantados: Tablas de Daimiel —en Castilla-La Mancha-, Picos de Europa -Asturias-; el programa del avellano en Cataluña; y el programa de estepas cerealistas de Castilla y León. Los seis obedecen a diferentes causas y en general tienen un trasfondo socioeconómico. Otros programas con regulación pero sin implantar son los de protección de áreas naturales y humedales característicos de Canarias, Aragón, Valencia y Murcia.

Sólo en Aragón, Baleares y Murcia se desarrollan los programas ZEPAS y Humedales RAMSAR en áreas específicas. La inversión entre 1993 y 1999 fue de 29.300 millones de pesetas, cuando el presupuesto era de cerca de 145.000 millones de pesetas. Es decir, una inversión efectiva inferior al 20\%, dedicada esencialmente a áreas cerealistas del interior.

\section{La política agroambiental a nivel de CA}

El nivel regional es una fase clave en el proceso de regulación e implantación de la política agroambiental. La mayor parte del programa 
agroambiental está regionalizado, en medidas horizontales (cada CA desarrolla la trasposición con una selección de medidas y su propio calendario) y en medidas zonales (reguladas por cada propia CA).

A este nivel de toma de decisión, el programa regional o zonal juega un papel clave, dado que determina la posición de los actores socioeconómicos e institucionales (nuevos y antiguos) en el proceso de regulación agroambiental. El juego de intereses entre diferentes actores influencia las medidas, presupuesto, límites geográficos y efectiva implantación. En estos programas zonales es posible analizar, por tanto, la convergencia o divergencia respecto a problemas, procesos y estructuras o planteamientos establecidos a nivel nacional. El programa zonal de mayor importancia, y más estrictamente agroambiental, es el denominado de Estepas de Castilla y León. Afecta a 512 municipios y 1,6 millones de ha, con un presupuesto aprobado de 30.000 millones de pesetas. Sus principales objetivos son: incrementar el set-aside, reducir la utilización de fertilizantes y reducir la producción mediante la re-introducción de prácticas agrarias extensivas consistentes en la conservación de los ecosistemas y del hábitat de la fauna silvestre en el área.

Administraciones públicas autonómicas y política agroambiental. Debido al carácter compartido de la gestión no es posible establecer una posición única de la Administración respecto al programa agroambiental. La administración agraria advierte que una buena parte de su actividad consiste en la prestación de ayudas públicas a los agricultores, lo que limita su capacidad de decisión. Las posibilidades de negociación respecto a la UE son reducidas. De esta forma, la administración agraria regional trata en muchos casos de 'dulcificar' y adaptar a las características de la agricultura regional una política negociada en otras esferas. Ello manifiesta un cierto grado de lejanía respecto a la política agraria 'de Bruselas', diseñada para agriculturas más competitivas e intensivas. Desde la administración agraria se reconoce la innecesariedad de desarrollo de políticas agroambientales (por ejemplo, algún año el barbecho financiado a los cultivos herbáceos por el régimen general de ayudas de la PAC fue superior al del programa agroambiental). Los problemas regionales de la agricultura están más relacionados con la sucesión en las explotaciones y la modernización de las mismas. Este llevar 'el papel de Bruselas a la realidad del campo' también se constata en el desarrollo del programa agroambiental, dado que muchas

$$
-335-
$$


de las medidas contenidas en los contratos agroambientales son excesivamente rígidas y escasamente operativas. En el plano más concreto del desarrollo del programa agroambiental de estepas se plantea que su financiación es suficiente para desarrollarlo, dado que en ningún año se ha agotado el presupuesto. El diseño de los límites del programa agroambiental se hizo de acuerdo a estudios de carácter ecológico y técnico, pero en el establecimiento de subzonas influyó el juego de intereses político. Respecto al desarrollo del programa se plantea una cierta incertidumbre en su continuidad o en su planteamiento actual al admitirse que los objetivos fijados para el mismo eran excesivamente ambiciosos, en lo que han influido actores externos al sector. El fracaso relativo obedece a una falta de difusión a nivel local ('el agricultor tiene muchas ayudas entre las que elegir') o a la inexistencia de un perfil claro del agricultor destinatario ('cada explotación es un mundo').

La administración ambiental gestiona una parte del programa, secundaria, pero de gran valor ecológico, al seleccionar parcelas con condiones idóneas para la reproducción de la avifauna. La participación de la administración ambiental supone un giro en la institucionalización de la política agraria regional, habitualmente llevada por la administración agraria y el cuerpo de agrónomos. La relación respecto a la administración agraria es escasa y existe independencia presupuestarioa ('son dos cajas diferentes'). Pero la separación se establece más allá de la propia gestión del programa. 'Aquí somos forestales y no agrarios' define un planteamiento y un punto de partida muy diferenciados, fundado en la adopción de 'la perspectiva ambiental y no agraria'. En todo caso se admite que este programa 'nos viene un poco de refilón', pero es adecuada para crear un 'mosaico de hábitat' para la avifauna característica de la zona. En consecuencia, la funcionalidad que se establece a la parte 'ambiental' del programa es estrictamente ecológica y no rural.

Sindicatos agrarios regionales y política agroambiental. Los sindicatos agrarios tienen unas posiciones generales coincidentes con sus correspondientes organizaciones de ámbito estatal. Las organizaciones de izquierda (UPA y COAG) son las que incentivan desde su inicio el programa agroambiental regional, utilizando argumentaciones de carácter ambiental, pero también social y económico. Se advierte que el desarrollo de la normativa agroambiental favorece al pequeño y mediano agricultor, y a todos aquellos que tienen explotaciones situadas en áre- 
as de rendimientos decrecientes. Desde esta perspectiva domina un planteamiento de carácter profesional, al entender las medidas agroambientales como una alternativa a las explotaciones marginales. En otro orden de cosas también aparece argumentaciones subyacentes a la ética tradicional del buen agricultor. Característica, esta última, que se comparte con las organizaciones de carácter empresarial. Para este último tipo de organizaciones toda política con un cierto carácter ambiental debe estar claramente separada de la política agraria, entendida como aquella que afecta a las rentas de los agricultores. De esta forma se defiende el carácter profesional y la competitividad de la agricultura en los mercados, constituyendo éste el eje del discurso.

En el plano más concreto del programa zonal de estepas existe, en cambio, una cierta alteración de los planteamientos más generales. Ambas orientaciones sindicales muestran una postura común sobre la necesidad d eque el programa agroambiental favorezca con exclusividad a agricultores profesionales. Asimismo se señala que una de las causas del escaso éxito del programa es el rechazo de los agricultores a la idea de 'agricultor-jardinero' y a los 'rollos medioambientales', aunque, en todo caso, si el agricultor debe realizar labores de conservación se sostiene que debe ser remunerado por ello. También existe unanimidad al indicar que para mejorar la aceptación de los contratos agroambientales por los agricultores éstos no deben introducir grandes modificaciones en su estrategia productiva y deben compensar en términos económicos. A este respecto se coincide en que existen demasiados condicionantes, tanto burocráticos como de gestión, en la fase de implantación, lo que condiciona el desarrollo del programa.

La evolución del programa agroambiental en Castilla y León: actores y territorio. El programa de zona de estepas cerealistas de Castilla y León es el primer programa de tipo agroambiental que se pone en marcha en España. Su proceso de implantación ha tenido diversas fases hasta su definitiva consolidación, en las que se afianzan determinados actores sociales, mientras que otros pierden relevancia (Paniagua, 1999).

1. ${ }^{a}$ fase. El intento de desarrollo de una política de Áreas Sensibles en zonas piloto. Durante los años 1991 y 1992, la UPA de Castilla y León suscita, ante la administración autonómica, la declaración de área ambientalmente sensible de las comarcas de Villafáfila (provincia de Za- 
mora) y Madrigal-Peñaranda (provincia de Salamanca) debido a su enorme riqueza de avifauna, y auspicia un acuerdo de colaboración con la Sociedad Española de Ornitología (SEO). Esta iniciativa, adoptada por la UPA regional, se integra en una estrategia de ámbito nacional de dicha organización sindical que pretende desarrollar en España las regulaciones ambientales establecidas hasta ese momento en el contexto de la PAC.

La figura comunitaria de Áreas Ambientalmente Sensibles, concebida para incentivar la conservación de aquellas zonas en las que la preservación de unos valores naturales sobresalientes depende del mantenimiento de las actividades agrícolas y ganaderas extensivas tradicionales, nunca había sido aplicada en España, en donde la implantación de medidas agroambientales ha sido muy reducida o ha estado ausente. La propuesta sobre las zonas de Villafáfila y Madrigal-Peñaranda era la primera iniciativa de este tipo sobre una área concreta.

El plan de gestión, diseñado en 1992 sobre un total de dos comarcas y 307.000 ha, que se propone por la Administración ambiental autonómica, incluye un conjunto de medidas con dos objetivos básicos: mantener o incrementar la población de aves esteparias, e incentivar mediante primas a los agricultores que establezcan contratos para mantener prácticas agrarias compatibles con el medio ambiente y la protección de las aves. la propuesta inicial de la Administración establece dos niveles (mantenimiento de actividades agrarias tradicionales y suprimir o limitar el uso de pesticidas y fertilizantes y, limitaciones relativas a la época de realización de determinadas prácticas agrícolas específicas) ligados directamente al grado de compromiso ambiental asumido por los agricultores. Este programa no se pone en marcha en 1992 por falta de presupuesto y de acuerdo entre las diferentes administraciones implicadas en su gestión y financiación (europea, estatal y autonómica). Esta falta de entendimiento propicia la adaptación a la normativa europea establecida en el nuevo reglamento 2078/92.

En esta fase, el principal protagonismo corresponde a la administración ambiental autonómica en menoscabo de la administración agraria, animada y auspiciada por las organizaciones agrarias neorrurales o de 'izquierdas', sobre todo los sindicatos UPA y COAG, al advertir que el desarrollo de estas medidas agroambientales pueden favorecer a su base social (pequeños y medianos agricultores), con la colaboración de algunas asociaciones de tipo conservacionista científico como la SEO. 
2. ${ }^{a}$ fase-1. ${ }^{a}$ etapa: Inicios del Programa de Estepas Cerealistas. En el mismo año 1992 la administración autonómica ambiental presenta a su discusión por las organizaciones agrarias el programa de zona en aplicación del nuevo reglamento 2078/92 sobre medidas agroambientales, en el que finalmente se incluirán las actuaciones propuestas para el programa piloto de Villafáfila y Madrigal (Programa, 1992). El programa zonal de estepas cerealistas es aprobado el 22 de julio de 1993 por la UE tras la reunión del comité STAR. El área se amplia considerablemente a 512 municipios y 1,7 millones de ha.

Este programa zonal tiene por objeto promover las prácticas agrarias compatibles con la conservación de las especies naturales y del hábitat de la avutarda en dos tipos de paisaje tradicional: Tierra de Campos y Tierra de Campiñas. En cada una de estas áreas se distinguen diversas subzonas.

Este programa se estructura en cuatro tipos de contrato con las siguientes características: los contratos 1 y 2 -ambos inspirados en los establecidos en el programa piloto de Villafáfila y Madrigal- implican aumento y mejora de la superficie de barbecho, mejora de pastos, reducción del nivel de fertilizantes, creación de bosquetes y linderas; el contrato 3 supone la retirada de tierras de la producción para fines ambientales y el contrato 4 la conservación de la diversidad biológica. Cada tipo de contrato tiene una aplicación en zonas delimitadas en función de la densidad de avutardas. Este programa presenta la singularidad de que los dos primeros contratos están gestionados por la administración agraria y los dos segundos por la administración ambiental. Este es el primer caso de una norma fruto de la PAC que es aplicada por un organismo no agrario en España. Además de los objetivos principalmente agroecológicos, el programa de estepas también incluye objetivos demográficos y sociales que persiguen mantener la densidad de población rural.

$2{ }^{a}$ fase-2. ${ }^{a}$ etapa: Consolidación del programa y reevaluación. Fruto de la presión de sindicatos agrarios de izquierdas (COAG y UPA), en noviembre de 1993 se promueve la primera convocatoria de ayudas dirigida a agricultores con tierras de secano incluidos en el ámbito del programa de zona y que adquieran el compromiso durante cinco años de realizar determinadas prácticas agrícolas. Se mantienen los cuatro tipos de contratos expuestos previamente con diversos condicionantes zonales en su gestión. 
El número de solicitudes durante el primer año de convocatoria ascendió a 275 afectando a una superficie de 24.637 ha. De ellas, 36 solicitudes y 1.595 ha se enmarcan dentro de los contratos 3 y 4 . De estas solicitudes se aprueban 224 con una superficie de 18.302 ha. Sólo 34 contratos y 620 ha se deben a los contratos 3 y 4 , gestionados por la administración ambiental. La propia administración considera reducida la aceptación del programa, sobre todo debido a la propia complejidad del mismo, como había sido puesto de manifiesto por las OPAs regionales.

Además, las modificaciones en el Reglamento UE 1765/92, que ampliaban el barbecho con derecho a prima hasta un $50 \%$ de la superficie de cultivo y establecía el incremento de su valor, provoca la desincentivación de la adopción de medidas agroambientales. Incluso generó la renuncia del 7,5\% de agricultores con contrato agroambiental.

En 1994 se modifica el programa con el fin de adaptarlo a la reglamentación general de ayudas a supeficies y hacer atractiva la adopción de medidas agroambientales entre los agricultores. Las modificaciones afectan fundamentalmente a los contratos 1 y 2 y pretenden conseguir mayor coherencia con las nuevas condiciones establecidas en el Reglamento 1765/92. La de mayor relevancia es el incremento de la superficie de barbecho. Con tales cambios se pretende agilizar el programa en lo referente a labores, simplificarlo en sus trámites burocráticos, coordinarlo con las ayudas por superficie a cultivos herbáceos y hacerlo atractivo económicamente. Ello conduce a un incremento de solicitudes dado que el barbecho exigido es equivalente al realizado con las ayudas a superficies de la PAC o el tradicional en la comarca. De esta forma el agricultor no precisa modificar profundamente la gestión habitual de la explotación. De nuevo se modifican parcialmente las condiciones del programa agroambiental para incentivar el número de beneficiarios, adaptando las condiciones 'impuestas por la UE' al modo de gestión habitual de la región.

Las repercusiones socioterritoriales han distado mucho de las previstas. La Administración autonómica había calculado una acogida del $50 \%$ de toda la superficie de aplicación del programa, cerca de 660.000 ha. En total no se han acogido más que el $18,3 \%$ de la superficie prevista y un $4,4 \%$ de las explotaciones (se preveía unas 40.000 explotaciones). Las causas de esta baja receptividad obedecen a problemas de difusión y adaptación profesional y social, a la división administrativa en su implantación, a la rigidez de los contratos frente a modificaciones cli- 
máticas y a la propia competencia de subvenciones de la UE. Los resultados han sido muy dispares entre tipos de contratos. Mientra que en el contrato 1 y 2 se han firmado 1.322 entre 1993 y 1997 afectando a 237 municipios y más de 120.000 ha. Con los contratos 3 y 4 que gestiona la administración ambiental se han establecido 248 contratos que abarcan 5.250 ha. Es decir, existe un claro desequilibrio entre tipos de contrato, dado que los de contenido más agrario ( 1 y 2 ) agrupan el $84.2 \%$ de los solicitantes y el $95,5 \%$ de la superficie. Las causas de esta disparidad son varias: el mayor tiempo de compromiso de los contratos 3 y 4 , que implican el no uso o la no comercialización de productos por veinte años; que estos contratos se dirigen a parcelas determinadas y no a explotaciones; y a un sistema de selección más riguroso, dado que la propia administración ambiental evalúa la aptitud ecológica de las parcelas aportadas a los fines ambientales del programa.

Por áreas geográficas y áreas establecidas en el programa agroambiental existe una mayor concentración en las provincias del Norte (Valladolid, León y Palencia) y tiene una menor intensidad en las provincias del Sur. La causa de este dispar reparto geográfico reside en la diferente estructura agraria. En las provincias del norte de la región las explotaciones son de tamaño medio-alto, mientras que en las meridionales tienen un tamaño más reducido. Ya los sindicatos agrarios habían apuntado que las condiciones de los contratos agroambientales favorecen más a las explotaciones de tamaño más elevado por la superficie de barbecho y la zona sin cultivar de los linderos. En consecuencia, el tipo de explotaciones que concurren a los contratos agroambientales son de tamaño elevado. El tamaño medio de los contratos 1 y 2 es de 92,7 ha, cuando el tamaño medio regional está en 20,5 ha y el $88 \%$ de las explotaciones regionales tiene menos de 50 ha. En los contratos 3 y 4 según los responsables de la administración ambiental no existe una relación directa entre tamaño de explotación y tipo de contrato.

$\mathrm{El}$ perfil de los agricultores que acceden a los contratos agroambientales ha ido cambiando paulatinamente, de acuerdo a las modificaciones introducidas en el programa. Existen dos tipos básicos de agricultores: agricultor a tipo principal y mayor de 50/55 años, sin sucesión, que accede al contrato agroambiental dado que le permite extensificar su explotación y 'tirar' hasta la edad de jubilación sin introducir modificaciones en la gestión; agricultor joven con una explotación grande, superior a 100 ha y con dedicación a tiempo parcial, en este caso 
se trata de agricultores bien situados económicamente y que no quieren cultivar o no pueden atender su explotación. También en abundantes ocasiones los agricultores son absentistas. Es decir, no residen en el municipio donde se ubica la explotación. En este sentido, la normativa agroambiental ha podido favorecer a agricultores no profesionales.

\section{El nivel local o de implantación: el discurso agroambiental entre los agricultores de Castilla y León}

El medio ambiente, la Naturaleza y la conservación de recursos son construcciones sociales que cada actor e incluso cada individuo entiende de diferente manera de acuerdo a la forma en que conceptualizan y organizan su mundo. De esta forma, las decisiones de los agricultores respecto a los programas agroambientales pueden estar influenciadas por múltiples factores (Brotherton, 1989, 1991). El discurso agroambiental entre los agricultores de Castilla y León se vertebra alrededor de tres ejes: el discurso ambivalente sobre la Naturaleza y el medio ambiente; la imagen de su responsabilidad profesional en el deterioro ambiental; y la introducción de nuevas medidas agroambientales.

Ruralidad y medio ambiente. Campo versus ciudad. Resulta complejo indagar en la imagen, desdoblada, que los participantes tienen del medio ambiente y de Naturaleza. La Naturaleza sería un conjunto armónico que incluiría a los seres vivos, a los recursos naturales, incluyendo a los agricultores. En cambio, la imagen del medio ambiente queda ligada, en este contexto, a la percepción del deterioro de la $\mathrm{Na}$ turaleza por la actividad humana y a la aparición de una creciente sensibilidad por los problemas ambientales y la búsqueda de soluciones. Esta sensibilidad ambiental presenta para los agricultores un carácter urbano. Queda enmarcada en el debate sobre las relaciones campo-ciudad y está asociada a la aparición o intrusión de nuevos actores sociales, principalmente ecologistas - urbanos- y administración ambiental -frente a la agraria_-, en la vida y organización de las áreas rurales.

Se pone de manifiesto que, para cada actor (visitante, ecologista, burócrata, agricultor), el medio ambiente presenta un significado característico. De aquí arranca la idea que esta variedad de concepciones sobre el medio ambiente no permite hacer coincidir los intereses de los 
diferentes grupos (como dicen ellos mismos, 'lo que es bueno para unos es malo para otros').

En concreto, se pone de manifiesto cómo la Administración mediante los programas agroambientales tiene una determinada concepción ambiental, fundada en la limitación y restricción de las prácticas agrícolas: 'lo quiere verde, sin cultivar, sin nada'. Otro grupo con una imagen definida del medio ambiente, a juicio de los agricultores participantes, son los grupos ecologistas, que tienen una visión claramente opuesta a la del agricultor profesional. Al quedar asociados al medio urbano y no al campo, se muestran críticos con sus actuaciones y consideran que priorizan en sus intereses al entorno ambiental frente a las personas (agricultores). Por contra, los agricultores plantean una visión del medio ambiente integrada en su actividad profesional y en el funcionamiento de la economía de las áreas rurales.

Medio ambiente y actividad profesional. Renta y cuidado del campo. El discurso del agricultor en relación a su actividad profesional se enmarca dentro del proceso de modernización de la agricultura, que arranca con la entrada en la Unión Europea y su integración en la Política Agraria Común y que se caracteriza por el desarrollo de una agricultura convencional, competitiva y profesional. En este contexto, los agricultores justifican sus intervenciones degradantes para el entorno en la necesidad y la obligación que tienen de producir para un mercado de masas. La rentabilidad en la producción es el elemento clave sobre el que se asienta esta justificación.

En esta parte del discurso de nuevo aparece el debate campo-ciudad de una forma conflictiva. El agricultor produce como demanda el hombre de la ciudad pero, de igual forma, el ecologista y la administración ambiental —asociados al discurso urbano sobre el campo- les plantean muchas limitaciones para desarrollar su actividad profesional. El agricultor se mueve entre estos dos ámbitos que percibe de forma conflictiva y contradictoria, lo que genera fuertes contradicciones profesionales, aunque asume la necesidad de conciliación sin articular fórmulas de conciliación adecuadas: «-Y tenéis los compradores de productos ecológicos como están en Francia, Alemania, en Italia y en esos países. Porque ahora, ¿a quién vendo yo ese producto ecológico? a nadie. Aquí, de la otra forma, tengo que echar herbicida para matar la semilla y joder el medio ambiente» (G. D. Santa María la Real de Nieva). 
Se propone la rearticulación del pacto social entre sociedad (administración) y agricultores, fundada en la transmisión de rentas por el cuidado del campo. «El medio ambiente, en cuanto haya dinero, funciona; los purines, en cuanto haya dinero, funciona; y funciona todo» (G. D. Santa María la Real de Nieva).

Pero, a su vez, el debate ambiental pone de manifiesto una cierta crisis de identidad profesional, fundada en la imagen del «buen agricultor» que es el que trabaja y vive exclusivamente en el campo, aún en un contexto de agricultura tecnificada y competitiva. Esta imagen es lo que les hace criticar a quien sólo cultiva para acogerse a las subvenciones. «Hacer las cosas bien» es seguir las reglas que, a su juicio, garantizan una buena producción, y el cuidado de la tierra para que produzca lo más posible.

La regulación ambiental y su incidencia en la actividad agraria en Castilla y León. La percepción por los agricultores de las políticas públicas de intervención en la agricultura ha sido un tema controvertido hasta el presente, dado que afecta tanto a su proceso de implantación, como a la toma de decisión de los poderes públicos (Battershill; Gilg, 1997).

a) La percepción de la política. La percepción de la política agroambiental o de los 'contratos agroambientales' es crítica y desfavorable. Sobre todo por la escasa rentabilidad económica de los contratos agroambientales respecto a otras subvenciones de la PAC: «Perdón, si fuera cincuenta mil pesetas la hectárea se apuntarían los propietarios, no se trabajarían las fincas, se quedarían baldías, entonces no habría producción, no se trabajarían las fincas" (GD Osorno).

De esta forma, el agricultor percibe que una modificación en las condiciones del programa, principalmente en sus aspectos económicos, condiciona la amplitud de sus resultados y, por tanto, implícitamente responsabiliza a los decisores públicos de su éxito o fracaso: «Depende de cada uno, de su explotación, de cómo éste y cómo tal y cómo cual. Entonces todo está estudiado, para que no vayamos al cien por cien y ¡pum! (GD Osorno).

Además de las estrictamente económicas, el agricultor percibe toda una serie de carencias en relación al programa agroambiental, principalmente relacionadas con el desarrollo y calendario muy rígido de las labores agrícolas: «Pero el nuestro empieza por ahí, porque tenemos 
que hacer unas labores en febrero, que es lo que hablábamos antes, esa no es mala, esa se puede, es asequible; pero después venimos lo de... para que nos siga, ya hemos comentado pero para que nos siga el orden, después venimos la de los abonos, esa se puede decir que es malísima; después está lo de la paja que el que tenga ganadería pues también es mala; y después está la hora de segar, de recolectar, que tenemos que recolectar muy tarde» (GD Villarín).

De esta forma parece que la política agroambiental es inflexible respecto a las posibles variaciones en el calendario agrícola, lo que se relaciona con la 'rigidez de Bruselas'. Además no se percibe un beneficio ambiental (reproducción de la avutarda) con la modificación de la distribución anual de las labores agrícolas: «Yo creo que eso ni lo pueden hacer, porque no tendrá nada que ver el tiempo para el cereal con el tiempo para los pollos, por ejemplo...» (GD Osorno).

Se concluye que la fase de toma de decisión y confección del programa está alejado de la realidad y deseos de los agricultores de la zona, los que deben implantar el programa y se constata la fractura entre los procesos de toma de decisión y de implantación, lo que contribuye a alimentar la sensación de abandono del agricultor profesional: «Perdona, referente a las estepas cerealistas, bueno pues es una modalidad que han sacado la Junta a través de Europa por potenciar un poquitico el medio ambiente, la fauna y el ecosistema de tal y el... de la avutarda. Y resulta que, bueno, pues que nos ponen todo trabas e incovenientes; porque, efectivamente, te dan un dinero que aparentemente es muy majo pero a costa de qué, si tienes que cumplir..., con la ley resulta que no interesa, no interesa por qué, porque si tienes que cumplir a rajatable como te marcan resulta que no se coge absolutamente nada» (GD Villarín).

Por tanto, las inconveniencias que se perciben del programa agroambiental radican tanto en la exigüidad de las subvenciones como en la alteración que supone de la gestión habitual de la explotación (calendario agrícola, exceso de barbecho, lindes, limitación al uso de herbicidas...).

b) La territorialización del programa agroambiental. En general se advierte que la territorialización del programa, tanto en su conjunto como en sus diferentes subzonas, obedece a razones de influencia política, local y regional, más que a cuestiones de tipo técnico. El diseño territorial del programa agroambiental induce a la percepción de agravios 
comparativos y al enfrentamiento territorial y social entre diversas comarcas de Castilla y León: «Sí, y lo solicité. No lo solicité de lleno porque me hicieron una jugada, que aquí estábamos con quince mil pesetas por hectárea para criar avutardas, pero como nos cambiaron de letra, pues Zamora es quien las cobra ahora. Hay tres letras A, B y C, nosotros estábamos en la A y ahí fue político, ahora todo es político» (GD Santa María la Real de Nieva).

El área de protección de la avutarda se podría reducir acusadamente si se hubieran seguido exclusivamente criterios ecológicos, al observarse que las avutardas tienden a concentrarse en unas zonas concretas, las de terrenos más arenosos, donde la producción agraria es más baja: «Y eso no sería más práctico dejar una parte del término.

- Te tienes que poner de acuerdo con todos.

-Bueno, pero yo es que lo veo más práctico, una zona de un término...

- Para mí que tendrían que ser zonas específicas.

- Hay que poner las que tiene ya cogidas el animal, que el animal mismo es el que las coge. Hay que estudiarlo y verlo. Que el animal es el que coge, el animal es muy inteligente... Y esas hay que cogerlas, donde está el animal.

- O sea que de dejar zonas para la avutarda tenían que ser zonas malas, que es donde le gusta a la avutarda vivir, en las zonas arenosas» (GD Santa María la Real de Nieva).

Se trata, en definitiva, de preservar de forma cuidadosa aquellas zonas de hábitat más intensivo del animal, áreas popularmente conocidas entre los vecinos de cada municipio. Con el actual sistema de territorialización no se consiguen los efectos deseados dado que junto a agricultores que realizan prácticas agroambientales hay otros que no las desarrollan.

c) La relación con la Administración. La implantación de programas agroambientales supone una modificación en la percepción de la Administración agraria, que habitualmente fomentaba la producción. Esta cede ante presiones de grupos o personas externas al sector - los ecologistas-, burocratiza en exceso la gestión del programa agroambiental, y forma parte del juego político para favorecer en las subvenciones a unas comarcas respecto a otras. La implantación de medidas de regulación agroambiental ha dado lugar a la intrusión en la toma de decisión de agentes a los que no se reconoce legitimidad — por su fal- 
ta de tradición- para abordar temas relacionados con las prácticas agrarias: «Si lo que hace falta es que cuando vienen los ecologistas que no nos vean a nosotros como detractores o como los camicazes del campo. Claro, porque somos personas que vivimos en él y muchas veces tenemos más intereses que ellos; lo que pasa que ellos se ciñen al papel y nos seve, a los mejor, que por dar un riego o por dar una cosa... se te vaya una cosecha» (GD Osorno).

Pero este discurso o sólo se organiza respecto a los ecologistas, sino también respecto a la propia administración ambiental que gestiona una parte del programa. De aquí es fácil pasar a un acusado victimismo, ligado a una percepción de abandono de lo agrario, de 'rendición' de la administración agraria.

La falta de entendimiento entre la Junta y la Unión Europea, o la lejanía de las directrices que impone esta última también han contribuido a acrecentar las dificultades de implantación.

d) El perfil del agricultor. El destinatario habitual de las medidas agroambientales es otro aspecto que aparece reiteradamente en el discurso de los agricultores. Las medidas que rigen el programa agroambiental son las que condicionan al posible destinatario. Pero, la discusión va más allá de qué tipo de agricultor es más beneficiado por las medidas, al contextualizarse en el debate sobre la desprofesionalización del agricultor. En general parece que las medidas de acompañamiento de la PAC benefician en mayor medida a agricultores no profesionales, en concreto a los agricultores jubilados o en la fase previa al retiro o con explotaciones que no tienen capacidad de cultivar adecuadamente. También interesa a propietarios absentistas y, por último, a los agricultores con explotaciones de bajo rendimiento: «- Luego, el que se agarra a todos esos beneficios no es el agricultor casi. El $90 \%$ no son agricultores, es que no son agricultores.

- No les interesa, van nada más a por pasta

- Es rentable para lagunas personas, pero para los demás no. (...)

- Para el que no viva del campo, para el señor que tanga otra renta en otra parte, porque el que vive del campo tiene que sacarle producción al campo.

— Lo más posible.

- ¿Claro!» (GD Santa María la Real de Nieva).

De igual forma se plantean ciertas repercusiones en el mercado de la tierra y en su movilidad. Dado que el sistema de subvenciones puede ha- 
cer que los propietarios absentistas retengan la tierra bajo su control o en caso de arrendarle eleven su renta. En cualquier caso, se apunta que las subvenciones sólo deberían ir dirigidas hacia quien es agricultor a título principal, sin que exista un claro acuerdo de qué características debe reunir esta figura.

\section{Conclusión}

La introducción de medidas de regulación ambiental en la agricultura europea se ha realizado sobre el principio de la subsidiariedad y el de la voluntariedad. Bajo el primero de estos principios los estados miembros han podido implantar o no estas normas en sus respectivos territorios, así como establecer sus propias normas, estándares, criterios y una determinada zonificación. El principio de voluntariedad opera a un nivel más micro. Un agricultor puede establecer (o no) de acuerdo a sus propias circunstancias e intereses (no sólo profesionales) las medidas agroambientales. Al establecerse una relación contractual entre administración y agricultores determinan primas de remuneración por actividades concretas, que el agricultor tiene una obligatoriedad plurianual de cumplir. De esta forma, la efectividad de las construcciones de política agroambiental quedan sujetas en último extremo a múltiples intereses individuales de los agricultores.

La normativa agroambiental comunitaria adopta un camino intermedio entre los clásicos acercamientos sobre implantación de políticas 'top down' y 'botton up', al posibilitar reordenar los principios europeos a cada nivel de toma de decisión, hasta el nivel local o de implantación. Fruto de ello resulta un proceso escalonado a distintos niveles o escalas con el fin de desarrollar una agricultura sustentable.

En España, este proceso se ha desarrollado a cuatro niveles y sobre tres esferas de negociación (europeo, nacional, regional y local). En los tres primeros prevalece la toma de decisión y en el último los problemas de implantación. La relación entre estos niveles tampoco jerárquica ni unidireccional, sobre todo entre los niveles nacional y regional.

La regulación 2078/92 establece un abanico de ayudas de carácter tanto agrario como rural. De éstas se adoptan un número reducido a nivel nacional, las de mayor contenido agrario. Además de dilatarse todo el proceso de trasposición. Ello coincide con la posición mantenida 
por el Ministerio de Agricultura, según el cual estas ayudas debían ser un 'comodín' a las rentas agrarias. En la propia negociación con Europa se impone un contenido más estrictamente ambiental.

El carácter cofinanciado de las medidas entre la UE, Estado y CCAA y el carácter regional de la competencia en agricultura y medio ambiente ofrecen como fruto un programa agroambiental nacional que representa una pluralidad de intereses de las CCAA, con escasas medidas de armonización entre ellas y un gran desarrollo de medidas zonales o regionales. España es el país de la UE que más programas presenta a financiación ante el comité STAR de la UE.

El peso regional del programa no ha impedido que España sea el país con menor número de programas zonales iniciados durante el primer cuatrienio de vigencia. Esta característica responde a que, dado el carácter cofinanciado del programa (UE-Estado-CA o UE-CA), la efectiva puesta en marcha viene determinada por el interés del programa agroambiental en los objetivos de política agraria de cada región, y la disponibilidad de fondos para tal fin. Se pone de relieve una escasa prioridad del programa agroambiental entre las CCAA. Por otra parte, se pone de manifiesto que cada CA gestiona y dirige su política agroambiental con escasa coordinación con otras CA.

Por otra parte, la efectiva implantación de estos programas ha supuesto una modificación en el estilo de implantación y la definitiva integración en una de las políticas de la PAC de agentes tradicionalmente secundarios y externos al sector agrario, incluida la administración ambiental. De igual manera, el ejemplo de Castilla y León pone de relieve que los factores relativos al programa son los que determinan el número y características de los agricultores que participan en el programa.

Para los agricultores, la introducción de medidas agroambientales representa un elemento más en el proceso de cambio de su función y en la desprofesionalización de su actividad. Además contribuye a desdibujar las tradicionales relaciones con la administración agraria. Para los agricultores participar o no en el programa depende principalmente de factores de tipo económico, aunque también del modo de gestión de sus explotaciones. Desde este nivel de implantación se advierte que los factores negativos del programa provienen de una toma de decisión alejada (Bruselas), sin tomar en consideración su adecuación a nivel nacional y regional. 


\section{BIBLIOGRAFÍA}

BALDOCK, D.; LowE, P., 1996: «The Development of European agri-environment policy», en Whitby, M. (ed.): The european environment and CAP reform. Policies and prospects for conservation. Walingford, CAB International, pp. 8-25.

BATTERSHILL, M. R. J.; GILG, A. W., 1997: «Socio-economic constraints and environmentally friendly farming in the southest of England», Journal of Rural Studies, 13, pp. 213-228.

BILlAUd, J. P., et al. (1997): «Social construction of the rural environment. Europe and discourses in France, Germany and Portugal», en HANN, H.; KASIMIS, B.; REDCLIFT, M. (eds.): Sustainable rural development, Aldershot, Ashgate, pp. 9-34.

BROTHERTON, I. (1989): «Farmer participation in voluntary land diversion schemes: some observations from theory», Journal of Rural Studies, 5, pp. 299-304.

- (1991): «What limits participation in ESAs?», Journal of Environmental Management, 32, pp. 241-249.

BULLER, H. J., 1992: "Agricultural change and the environment in Western Europe», en HoGGART, K. (ed.), Agricultural change, environment and economy. Essais in honour of W. B. Morgan. Londres, Mansell, pp. 68-88.

COMISIÓN EUROPEA, 1996: La situación de la agricultura en la Unión Europea, Bruselas, UE.

CLARK, J. R. A.; JONES, A., 1998: «Agricultural élites, agarian beliefs, and their impact on the evolution of agri-environment policies: an examination of the British experience, 1981--92», Environment and Planning A, vol. 30, pp. 2227-2243.

ClARK, A., et al., 1997, Conceptualising the evolution of the European Union's agri-environment policy: a discourse approach, Environment and Planning A, 29, 1869-1885.

CURTIS, A.; DE LACEY, T., 1991: «Landcare, stewardship and sustainable agriuclture in Australia», Environmental Values, vol. 7, n. ${ }^{\circ}$ 1, pp. 59-78.

DeverRe, CH., 1995, «Social implications of agro-environmental policy in France and Europe», Sociologia Ruralis, vol. XXXV, n. ${ }^{\circ}$ 2, pp. 227-247.

GARRIDO, F.; MoYANO, E., 1996, «Spain», en WhitBY, M., The european environment and CAP reform. Policies and prospects for conservation. Wallingford, CABI, 86-104 pp.

GuBLIELmi, M., 1995: «Vers de nouvelles fonctions de l'agriculture dans l'espace?», Economie Rurale, n. $^{\circ} 229$, sept-oct, pp. 22-28.

HOGGART, K.; BULLER, H.; BLACK, R., 1995: Rural Europe. Identity and change. Londres, Arnold, 319 pp.

ILBERY, B., 1992: «State-assited farm diversificacition in the United Kingdom», en BowLER, I. R.; BRYANDT, C. R.; NELLIS, M. D. (eds.), Contemporary rural systems in transition, vol. 1. Agriculture and environment, Londres, CAB Int., 100-116.

LOWE, P.; WARD, N., 1998, «Regional policy, CAP reform and rural development in Britain: the challenge for new labour», Regional Studies, vol. 32.5, pp. 469-479.

Mrtchell, B., 1989, Geography and resource analysis. New York, Longman, 386 pp.

PANIAGUA, A., 1997, «Significación social e implicaciones para la política agraria de la 'cuestion ambiental' en el medio rural español», en GómEz, C.; GonzÁLEz, J. J., Agricultura y Sociedad en la España Contemporánea, Madrid, CIS-MAPA, pp. 975-1015.

PANIAGUA, A., et al., 1998: «Análisis de la normativa agroambiental comunitaria, estatal y autonómica en el período de 1985 a 1997», REE. Revista de Estudios Europeos, n. ${ }^{\circ} 18$, pp. 51-70.

PANIAGUA, A., 1999: «Cambio rural y política agroambiental. El caso del programa de estepas cerealistas de Castilla y León", Anales de Geografía de la Universidad Complutense, 19, pp. 169-189.

POTTER, C., 1998: Against the grain. Agri-environmental reform in the United States and the European Union. Wallingford, CABI, $194 \mathrm{pp}$. 
PotTeR, C.; Goodwin, P., 1998: «Agricultural liberalization in the European Union: an analysis of the implications for nature conservation», Journal of Rural Studies, vol. 14, n. ${ }^{\circ}$ 3 , pp. 287-298.

MURDOCH, J.; WARD, N., 1997: «Govermentality and territoriality: the statistical manufacture of Britain's national farm», Political Geography, 16, pp. 307-324.

Programa de zona de aplicación del Reglamento 2078/92 en las Estepas Cerealistas de Castilla y León. Valladolid, Junta de Castilla y León, 1992, mec.

Regina SegurA, A., 1996: «Reflexiones sobre el Reglamento Agroambiental europeo y el programa nacional español», Quercus, 125, pp. 46-48.

SeCretaría General de Estructuras Agrarias, 1994, Programas de ayudas para fomentar métodos de producción agraria compatibles con las exigencias de la protección y la conservación del espacio natural. Madrid, MAPA (mimeo) 3 vols.

WARD, N., et al., 1998, «Keeping matter in its place: pollution regulationand the reconfiguring of farmers and farming», Environment and Planning A, vol. 30, pp. 1165-1178.

WILSON, G. A., 1995: «German agri-environmental schemes- II. The MEKA programme in Baden-Württemberg», Journal of Rural Studies, vol. 11, n. ${ }^{\circ} 2$, pp. 149-159.

wilson, G.; Bryant, R., 1997: Environmental management. New directions for the twenty-first century, Londres, UCL, $202 \mathrm{pp}$.

RESUMEN: La legislación agroambiental de la Unión Europea especifica que cada estado miembro puede determinar sus propias normas, definir estándares y designar áreas. Un resultado de esta flexibilidad es que cada Estado Miembro puede adaptar y completar la regulación básica. Ello ha producido que el modelo de implantación de las medidas agroambientales en cada país responda a intereses de distintos actores sociales.

El desarrollo de la política agroambiental en España responde a las directrices de la UE y al juego de intereses de la CCAA. Con antelación a la regulación agroambiental, la experiencia española en normativa agroambiental prácticamente era inexistente. De las medidas de acompañamiento de la nueva PAC de 1992, los agroambientales son las últimas en verse introducidas en España. A nivel nacional el programa agroambiental nacional sólo utiliza dos de las siete medidas mencionadas en la regulación de la UE. Las otras cinco medidas de la regulación 2078/92 quedan recogidas en los programas regionales y son aplicadas en áreas geográficas específicas. En términos de énfasis vertical y horizontal, España queda a medio camino en el proceso de implantación de medidas agroambientales. El programa nacional toma carta de naturaleza en 1995. Pero las medidas regionales se ha desarrollado de forma desigual. Sólo seis de los 66 programas aprobados han sido desarrollados e implantados a finales de 1998. A nivel local la efectividad de las medidas regionales es reducida debido a factores relativos a la propia confección de los programas (falta de coordinación política, competencia financiera con otras medidas de la PAC) y debido a factores actitudinales (escepticismo de los agricultores y de la propia administración para su implantación).

Palabras Claves: Política agroambiental, toma de decisión, agricultores, niveles de decisión, actores sociales, España.

ABSTRACT: EU agri-environmental legislation specifies that every Member State may determine its own norms, specifying standards and designated areas. One result of this flexibility for Member States is that there is substantial scope for basic principels to be adapted and completed in every country. This give rise to very different interests being expresed by different social actors and to very varied methods of implementation for agrienvironmental measures. 
The development of environmental policy in Spain has been balanced, on one hand to joining the EU and, on the other hand to the interests of political autority to Spain's regions. Prior to EU agrienvironmental regulations, the Spanish experience of introducing environmental considerations into farm production was practically non-existent. Thus, of the new CAP accompanying measures, the agrienvironmental ones have been the last to be introduced into Spanish agriculture. At the national level the spanish agrienvironmental programme only uses two of the seven types mentioned by EU regulation. The other five measures of the regulation 2078/92 are incorporated in regional programmes for aid applied to specific geographical areas in accordantce with special environmental sensitivities. In therms of the horizontal or vertical emphasis, Spain is in a mid-way situation in the way it has implemented agri-environmental measures. The national basis were put in effect in 1995. But the regional measures had developed in inequality terms. Only 6 of 66 programmes has developed and implemented at the end of 1998. At the local level the effectivity of regional measures are reduit for scheme factors (the lack of policy coordination, the competition from other CAP financial measures) and the actitudinal factors (scepticism of farmers and reluctance from the agricultural autorities).

KEY wORDS: agrienvironmental policy. Decision-makers, farmers, levels of decisions, actors, Spain. 